\title{
Irbesartan Ameliorates Hyperlipidemia and Liver Steatosis in Type 2 Diabetic db/db Mice via Stimulating PPAR- $\gamma$, AMPK/Akt/mTOR Signaling and Autophagy
}

\author{
Juan Zhong ${ }^{\mathrm{a}, \mathrm{b}, \mathrm{d}}$, Wangqiu Gong ${ }^{\mathrm{b}}$, Lu Lu ${ }^{\mathrm{a}, \mathrm{b}}$, Jing Chen ${ }^{\mathrm{c}}$, Zibin Lu ${ }^{\mathrm{a}}$, HongYu Li ${ }^{\mathrm{b}}$, \\ Wenting Liu ${ }^{\mathrm{b}}$, Yangyang Liu ${ }^{\mathrm{a}, \mathrm{b}}$, Mingqing Wang ${ }^{\mathrm{a}, \mathrm{b}}$, Rong Hu${ }^{\mathrm{a}, \mathrm{b}}$, Haibo Long ${ }^{\mathrm{a}, *}$, \\ Lianbo Wei ${ }^{\mathrm{a}, \mathrm{b}, *}$
}

Key words: Irbesartan, Hyperlipidemia, Liver steatosis, PPAR- $\gamma$, AMPK/Akt/mTOR signaling, Autophagy

\begin{abstract}
Irbesartan (Irb), a unique subset of angiotensin II receptor blockers (ARBs) with PPAR- $\gamma$ activation function, has been reported to play a role in renal dysfunction, glucose metabolism, and abnormal lipid profile in diabetic animal models and humans. However, the underlying mechanisms that improve hyperlipidemia and liver steatosis are unclear. This study investigated the effects of Irb on lipid metabolism and hepatic steatosis using the spontaneous type 2 diabetic $\mathrm{db} / \mathrm{db}$ mouse model. The results demonstrated body and liver weight, food consumption, lipid content in serum and liver tissue, and liver dysfunction as well as hepatic steatosis were increased in $\mathrm{db} / \mathrm{db}$ mice compared with $\mathrm{db} / \mathrm{m}$ mice, whereas the increases were reversed by Irb treatment. Moreover, Irb administration resulted in an increase in LC3BII as well as the LC3BII/I ratio through activating PPAR- $\gamma$ and $p$-AMPK and inhibiting $p$-Akt and p-mTOR, thereby inducing autophagy in the $\mathrm{db} / \mathrm{db}$ mouse liver. Therefore, our findings suggest that Irb can ameliorate hyperlipidemia and liver steatosis by upregulating the expression of PPAR- $\gamma$, activating the AMPK/Akt/mTOR signaling pathway and inducing liver autophagy.
\end{abstract}

\section{Introduction}

Hyperlipidemia is considered to be a crucial risk factor in patients with type 2 diabetes. Along with hyperlipidemia and abnormalities in blood glucose, diabetes 
leads to micro- and macro-vascular complications, which are the major causes of morbidity and death in diabetics[1,2]. Meanwhile, the excess deposition of triglycerides (TG) and/or total cholesterol (TC) in the liver causes liver steatosis, which is the initial stage of non-alcoholic fatty liver disease (NAFLD) [3]. If uncontrolled, hepatic steatosis will progress to life-threatening diseases, such as hepatic fibrosis and dysfunction. Moreover, recent data increasingly support a complicated interplay between the metabolic condition of diabetes mellitus (DM) and NAFLD. In diabetic patients, increased free fatty acids (FFAs) in the liver accelerate the progression of fatty liver to steatohepatitis [4,5]. Irbesartan (Irb), an angiotensin II receptor blocker (ARB), acts as a selective peroxisome proliferator-activated receptor- $\gamma$ (PPAR- $\gamma$ ) agonist and thus may have anti-inflammatory and antioxidative functions, as well as beneficial effects on glucose and lipid metabolism [6]. However, the underlying mechanisms that improve hyperlipidemia and liver steatosis under the metabolic condition of DM are not clear.

Autophagy is crucial for development, differentiation, homeostasis, and survival and has been shown to play important roles in ameliorating lipid metabolism and hepatic steatosis [7]. Impaired autophagy decreases hepatic fatty acid $\beta$-oxidation and TG output, thereby inducing liver steatosis. Additionally, liver steatosis is ameliorated when hepatic autophagy is provoked by certain compounds or signaling pathways [3]. Moreover, Singh et al. [8] showed that the interrelationship of autophagy and lipid metabolism may trap hepatocytes in a harmful cycle in which reduced autophagy promotes lipid accumulation that then further restrains autophagic function, thereby additionally exacerbating lipid retention. In addition, it has been reported that mice with chronic obesity or insulin resistance that are susceptible to NAFLD have obviously decreased hepatic autophagy indicators [3,7].

$\mathrm{Db} / \mathrm{db}$ mice, a genetic model of obesity and insulin resistance resulting from a leptin receptor mutation, can spontaneously exhibit hyperglycemia, hyperlipidemia, and hepatosteatosis, which is similar to steatosis in human beings [9,10]. Current research has shown that the protein level of LC3BII is decreased in the later stages of diabetic mice and that the impairment of autophagy in the liver plays an important role in aggravating lipid metabolic disorders and contributing to steatohepatitis [3,4]. Therefore, we determined the possible role of autophagy in the beneficial effects of Irb on lipid metabolism and hepatic steatosis in diabetic $\mathrm{db} / \mathrm{db}$ mice.

\section{Materials and Methods}

\subsection{Animals and treatment groups}

Ten-week-old male and female $\mathrm{db} / \mathrm{db}$ mice on a C57BL/KsJ (BKS.Cg-Dock7m +/+ Leprdb/J) background and age-matched wild-type $\mathrm{db} / \mathrm{m}$ littermates were purchased from the Animal Model Research Center of Nanjing University. After 1 week of acclimatization, the $\mathrm{db} / \mathrm{db}$ mice were randomly divided into 2 groups $(\mathrm{n}=12$ each, with equal halves male and female mice): $\mathrm{db} / \mathrm{db}$ mice and $\mathrm{db} / \mathrm{db}$ mice+Irb.

Additionally, $12 \mathrm{db} / \mathrm{m}$ mice, half male and half female, were used as the control group. 
After 16 weeks of Irb treatment, the mice were fasted overnight and sacrificed for the experiment. All mice were housed in the Laboratory Animal Center of Southern Medical University under controlled temperature $\left(22-25^{\circ} \mathrm{C}\right)$, humidity $(60 \%)$, and light (alternating 12-hour light/dark cycle) conditions with free access to food and water. The animals were fed standard rodent chow, which contained $<0.1 \%$ cholesterol and $13.4 \%$ of the total calories from fat (Keao Xieli Feed Co. Ltd., Beijing, China; No. KA3211). The amount of food intake per mouse was calculated as the decrease of supplied food weight every day, and changes in the weekly food intake were compared among the three groups. All study protocols were reviewed and approved by the Animal Care and Use Committee of Southern Medical University and were in accordance with the National Institutes of Health Guidelines of Laboratory Animal Care and Use (Publication number 85-23, revised 1985).

\subsection{Irb administration}

Irb was provided by Sanofi-Aventis Pharmaceuticals Company (Hangzhou, China; Batch No. 5A173) and was made fresh daily by dissolving a small amount in normal saline. Irb was administered to mice daily via oral gavage at a dose of $50 \mathrm{mg} / \mathrm{kg}$ for 16 weeks; this dose corresponded to the dose of Irb used in humans [11], which was calculated and converted to $\mathrm{mg} / \mathrm{kg}$ in our preliminary study as well as in previous reports using a mouse model [12-15]. Mice in the control group and the $\mathrm{db} / \mathrm{db}$ model group received an equivalent volume of saline solution.

\subsection{Serum measurements}

At the end of the animal experiments, mice were anesthetized; then, blood was obtained from the eyes of the mice. Serum TG, TC, aspartate aminotransferase (AST), and alanine aminotransferase (ALT) levels were measured using an automatic analyzer (Roche, Basel, Switzerland). Fasting blood glucose (FBG) was detected at 0 , $4,8,12$, and 16 weeks of Irb treatment using a glucose meter (Roche Diagnostics $\mathrm{GmbH}$, Mannheim, Germany).

\subsection{Tissue lipid contents and histological analysis}

The liver and adipose tissue were removed, rinsed with physiological saline, and immediately weighed. The hepatic FFA, TC and TG contents were measured using commercial kits. (Jiancheng, Nanjing, China). Tissue samples were stored at $-80^{\circ} \mathrm{C}$ until analysis.

The liver was fixed with $10 \%$ neutral formalin; subsequently, the fixed tissue was embedded in paraffin and 3-5 $\mu \mathrm{m}$ sections were cut and stained with hematoxylin and eosin (HE). The severity of NAFLD was evaluated semi-quantitatively using the NAFLD activity score (NAS) system as follows [16-18]: 0, steatosis less than 5\%; 1, steatosis between $5 \%$ and $33 \%$; 2 , steatosis between $33 \%$ and $66 \%$, and 3 , steatosis greater than $66 \%$. Liver steatosis included hepatocellular hypertrophy, microvesicular steatosis, and macrovesicular steatosis; therefore, the NAS score was the sum of the scores of these pathological changes. The frozen sections were stained with Oil Red O 
solution to further verify lipid droplet accumulation, and areas positive for Oil Red O were quantified using Image-Pro Plus 6.0 software (IPP, Media Cybernetics, Inc., USA).

\subsection{Western blot analysis}

Protein from each liver sample was extracted with lysis buffer at $4^{\circ} \mathrm{C}$. Then, the extracts were centrifuged at $12,000 \mathrm{rpm}$ and $4^{\circ} \mathrm{C}$ for $30 \mathrm{~min}$, and the supernatants of these tissues were used for Western blotting analyses. The protein samples were separated on $10 \%$ SDS-polyacrylamide gels and subsequently transferred onto PVDF membranes (Millipore, Bedford, MA). The membranes were blocked with 5\% nonfat milk in TBST (20 mM Tris-HCl, $\mathrm{pH} 7.6,0.9 \% \mathrm{NaCl}, 0.1 \%$ Tween 20) for $1 \mathrm{~h}$ at room temperature and then incubated with primary antibodies overnight at $4{ }^{\circ} \mathrm{C}$. After washing in TBST, the membranes were exposed to the secondary antibodies for $2 \mathrm{~h}$ at room temperature. Antibodies specific for PPAR- $\gamma$, adenosine monophosphate-activated protein kinase (AMPK), p-AMPK, Akt, p-Akt (S473), LC3B, and $\beta$-actin were obtained from Cell Signaling Technology (Danvers, USA). Antibodies against mTOR, p-mTOR (S2448), leptin, leptin receptor (LepR), and PPAR- $\alpha$ were purchased from Abcam (Cambridge, UK). The target proteins were detected and analyzed using a CCD system (Image Station 2000 MM, Kodak).

\subsection{Immunohistochemistry (IHC)}

The liver sections $(3 \mu \mathrm{m})$ were dewaxed in xylene and rehydrated through a graded series of ethanol concentrations. Then, the slides were pretreated with a microwave antigen retrieval technique and blocked with 5\% BSA. The slices were incubated with an anti-LC3B monoclonal antibody $(1: 500)$ overnight at $4^{\circ} \mathrm{C}$, followed by incubation with a secondary goat anti-rabbit antibody for $30 \mathrm{~min}$ at $37^{\circ} \mathrm{C}$. After washing, the DAB chromogen was added for 2 min before counterstaining with hematoxylin. Sections were observed with an optical microscope (Ni-U, Nikon Corporation, Tokyo, Japan), and images were analyzed with IPP software.

\subsection{Immunofluorescence (IF)}

To further assess the expression of LC3B protein in liver tissue, fresh mouse liver tissue was fixed with $4 \%$ paraformaldehyde for $1 \mathrm{~h}$ on ice and dehydrated in $20 \%$ sucrose in PBS overnight at $4{ }^{\circ} \mathrm{C}$, and the sections $(5 \mu \mathrm{m})$ were then prepared with a freezing microtome. Subsequently, cryosections were dried for $30 \mathrm{~min}$ at room temperature, permeabilized in PBS containing 0.1\% Triton X-100 for $15 \mathrm{~min}$, blocked with $2 \%$ BSA for $1 \mathrm{~h}$, and washed three times with cold PBS for $5 \mathrm{~min}$. The sections were incubated overnight with anti-LC3B antibody $(1: 50)$ at $4^{\circ} \mathrm{C}$, followed by incubation with goat anti-rabbit IgG conjugated with $\mathrm{Cy} 3$ from Jackson ImmunoResearch Laboratories (Baltimore, USA) for $1 \mathrm{~h}$ at room temperature. After washing with PBS, DAPI was used to counterstain the cell nuclei for $15 \mathrm{~min}$. Images were obtained with a Zeiss (Jena, Germany) LSM710 confocal microscope and analyzed using IPP software. 


\subsection{Transmission electron microscopy (TEM)}

Liver tissue from each group was fixed with 2.5\% glutaraldehyde, washed in PBS, and post-fixed in $1 \%$ osmium tetroxide. Then, the samples were dehydrated by serial dilutions of ethanol, embedded in EPON resin, and stained with uranyl acetate and lead citrate. Finally, images were viewed and captured by a transmission electron microscope (HITACHI H-7650, Tokyo, Japan).

\subsection{Statistical analysis}

Data are shown as the means \pm standard deviations (SD). Statistical significance was analyzed using one-way ANOVA, followed by either a least significant difference (LSD) test or Dunnett's T3 test for multiple comparisons, based on a homogeneity of variance test conducted using SPSS 13.0 (SPSS, Chicago, IL). Differences were considered significant at a $P$ value $<0.05$.

\section{Results}

3.1 Food intake and body and tissue weights

All mice were fed a standard chow diet; however, db/db mice had a significant increase in total food intake compared with that of $\mathrm{db} / \mathrm{m}$ mice. Furthermore, this increase was reversed by Irb as shown in Table 1. Moreover, at the end of the experiment, the $\mathrm{db} / \mathrm{db}$ mice had significantly greater body weight, liver weight, and epididymal and subcutaneous adipose tissue weights than $\mathrm{db} / \mathrm{m}$ mice (Table 1 ). After 16 weeks of treatment with Irb, the body weight and liver weight expressed as a percentage of body weight in the $\mathrm{db} / \mathrm{db}$ mice+Irb group was significantly decreased compared with the $\mathrm{db} / \mathrm{db}$ mice group (Table 1 ). Irb tended to suppress subcutaneous and epididymal fat weight gain in $\mathrm{db} / \mathrm{db}$ mice, but the difference was not statistically significant (Table 1).

3.2 Irb improved the serum parameters of the lipid profile, FBG and liver function The FBG was detected after $0,4,8,12$, and 16 weeks of Irb treatment, and, compared with $\mathrm{db} / \mathrm{m}$ mice, the FBG was significantly increased in $\mathrm{db} / \mathrm{db}$ mice (Figure 1A). Moreover, FBG was dramatically increased in older $\mathrm{db} / \mathrm{db}$ mice but remained stable in $\mathrm{db} / \mathrm{m}$ mice (Figure 1A); however, Irb markedly slowed this sharp FBG increase in $\mathrm{db} / \mathrm{db}$ mice, especially after 12 weeks of Irb administration and beyond (Figure 1A). Serum TG and TC levels were significantly elevated in $\mathrm{db} / \mathrm{db}$ mice compared with $\mathrm{db} / \mathrm{m}$ mice (Figure 1B), and two representative indexes of liver function (ALT and AST) were also higher in $\mathrm{db} / \mathrm{db}$ mice (Figure 1C), indicating macrovesicular and microvesicular steatosis-induced liver injury (as confirmed by Oil Red O and HE staining). In contrast, the Irb-treated db/db mice showed a significant decrease in the serum lipid profiles (TG and TC), and liver function indexes (ALT and AST) as illustrated in Figure 1B and 1C.

3.3 Irb alleviated lipid contents and pathological changes in the liver Consistent with the serum results, the hepatic TG, TC, and FFA levels were significantly higher in $\mathrm{db} / \mathrm{db}$ mice than in $\mathrm{db} / \mathrm{m}$ mice, and Irb administration resulted 
in a distinct reduction in hepatic lipid contents (TG, TC, and FFA) as shown in Figure 2A and 2B. Additionally, the lipid metabolism data as well as the body and tissue weight gains were separately analyzed in female and male $\mathrm{db} / \mathrm{db}$ mice, and the results were similar to those of the general analysis (Supplemental Tables 1 and 2, Supplemental Figure 1). The $\mathrm{db} / \mathrm{db}$ mice showed an increase in lipid accumulation and pronounced steatosis in the liver, which was characterized by prominent ballooning injury, vacuolation, and hepatocellular hypertrophy, etc. (Figure 2C). Moreover, the NAS score was remarkably higher in $\mathrm{db} / \mathrm{db}$ mice than in $\mathrm{db} / \mathrm{m}$ mice, and this increase was inhibited by Irb (Figure 2E). In addition, Oil Red O staining further confirmed the beneficial effects of Irb on hepatic histology by reducing the accumulation of fatty droplets (Figure 2D and 2F).

\subsection{Irb decreased the leptin levels and increased the expression of PPAR- $\alpha$ in tissues} $\mathrm{Db} / \mathrm{db}$ mice have a point mutation in LepR, which manifests as severe leptin resistance and LepR deficiency. Therefore, compared with $\mathrm{db} / \mathrm{m}$ mice, these mice exhibit significantly increased leptin levels and obviously decreased LepR levels in the liver, brown fat and heart. Irb treatment remarkably decreased leptin expression and ameliorated leptin resistance in tissues, but the expression levels of LepR in the tissues were not significantly different between the $\mathrm{db} / \mathrm{db}$ mice group and $\mathrm{db} / \mathrm{db}$ mice+Irb group (Supplemental Figure 2). Additionally, PPAR- $\alpha$ is a nuclear receptor of ligand-activated transcription that can stimulate lipid consumption by enhancing the expression of genes that oxidize fatty acids. We also found that the PPAR- $\alpha$ levels were lower in $\mathrm{db} / \mathrm{db}$ mice than in $\mathrm{db} / \mathrm{m}$ mice, whereas Irb administration resulted in a distinct increase in the PPAR- $\alpha$ expression in relevant tissues (Supplemental Figure 2).

3.5 Irb elevated PPAR- $\gamma$ levels and activated AMPK/Akt/mTOR signaling in the liver Irb treatment can upregulate the expression of PPAR- $\alpha$ in the tissues of $\mathrm{db} / \mathrm{db}$ mice; however, Irb is a known PPAR- $\gamma$ agonist, but its ability as a PPAR- $\alpha$ agonist requires further elucidation. Moreover, PPAR- $\gamma$ is also a member of the nuclear receptor superfamily and is an important regulator of lipid and glucose homeostasis; these processes control lipid mobilization into adipocytes by promoting adipogenesis and regulating the expression of adipocyte-secreted proteins and adipocytokines such as leptin and adiponectin, thereby reducing lipotoxicity [19,20]. In db/db mice, the level of PPAR- $\gamma$ protein was dramatically decreased, but Irb treatment significantly increased the protein expression of PPAR- $\gamma$ as shown by Western blot analysis (Figure 3A and 3B). Interestingly, Irb also increased AMPK expression levels, promoted AMPK phosphorylation, and reduced the phosphorylated form of Akt, which contributed to the suppression of mTOR phosphorylation in $\mathrm{db} / \mathrm{db}$ mouse livers, as shown in Figure 3C and 3D. Therefore, these results suggest that Irb can regulate the expression of p-AMPK, p-Akt (Ser473) and p-mTOR (S2448), thereby triggering the related biological effects of the AMPK/Akt/mTOR signaling pathways. To further confirm this conclusion, we detected the expression of the autophagosome marker LC3B using Western blotting, IHC and IF in liver tissue from each group. 
3.6 Irb induced autophagy in the livers of $\mathrm{db} / \mathrm{db}$ mice

Compared with $\mathrm{db} / \mathrm{m}$ mice, $\mathrm{db} / \mathrm{db}$ mice had an obviously decreased level of IHC staining for LC3B, as demonstrated in Figure 4B and 4D. Importantly, when db/db mice were treated with Irb for 16 weeks, the expression of LC3B was significantly increased. Furthermore, the results of IF staining were consistent with the IHC results, which revealed that the expression of LC3B was reduced in the liver tissue of $\mathrm{db} / \mathrm{db}$ mice, but the effects were reversed by Irb (Figure 4A and 4C).

To further determine the effect of Irb on autophagy in the livers of $\mathrm{db} / \mathrm{db}$ mice, the protein expression of LC3B was assessed. We found that the protein level of LC3BII (the localized form in the autophagosomal membrane) and the LC3BII/I ratio (an autophagy activity marker) were decreased in $\mathrm{db} / \mathrm{db}$ mice. However, Irb treatment induced an increase in LC3BII as well as the LC3BII/I ratio, which was decreased in the liver tissue of $\mathrm{db} / \mathrm{db}$ mice (Figure $4 \mathrm{E}$ and $4 \mathrm{~F}$ ).

In our study, the presence of autophagosomes was detected by TEM, which was also used to identify the autophagic process. Vacuoles with free membrane structures and typical double membranes were observed in the cytoplasm in each group, as shown by the red arrowheads in Figure 4G. The TEM results suggested a tendency towards a decreased number of autophagosomes in $\mathrm{db} / \mathrm{db}$ mice compared with $\mathrm{db} / \mathrm{m}$ mice, whereas the number of autophagosomes was elevated with Irb treatment. Overall, our findings indicate the potential mechanisms regulating how Irb alleviates hyperlipidemia and liver steatosis, as we show that the drug elevated PPAR- $\gamma$ levels, activated the AMPK/Akt/mTOR signaling pathway, and induced autophagy in the livers of $\mathrm{db} / \mathrm{db}$ mice.

Table 1: The effects of Irb on food intake, body weight and tissue weight

\begin{tabular}{cccc}
\hline & $\mathrm{db} / \mathrm{m}$ & $\mathrm{db} / \mathrm{db}$ & $\mathrm{db} / \mathrm{db}+\mathrm{Irb}$ \\
\hline Food intake (g/day) & $2.98 \pm 0.19$ & $6.25 \pm 0.83^{*}$ & $5.31 \pm 0.60^{* \#}$ \\
Body weight (g) & $23.51 \pm 2.75$ & $53.41 \pm 4.36^{*}$ & $42.87 \pm 6.44^{* \#}$ \\
Liver weight (\% BW) & $5.19 \pm 0.40$ & $8.15 \pm 1.47^{*}$ & $6.82 \pm 0.83^{* \#}$ \\
Epididymal fat (\% BW) & $1.36 \pm 0.38^{\#}$ & $6.94 \pm 0.91^{*}$ & $5.99 \pm 1.11^{*}$ \\
Subcutaneous fat (\% BW) & $1.58 \pm 0.55^{\#}$ & $13.37 \pm 4.29^{*}$ & $10.03 \pm 1.54^{*}$ \\
\hline
\end{tabular}

The data are expressed as the means $\pm \mathrm{SD} ; \mathrm{n}=12$ per group (except for epididymal fat; $\mathrm{n}=6$ ). ${ }^{*} P<0.05$ versus $\mathrm{db} / \mathrm{m} .{ }^{\#} P<0.05$ versus $\mathrm{db} / \mathrm{db}$.

Figure 1: Irb alleviates dysregulated blood lipid and FBG levels as well as liver dysfunction. (A) The FBG levels were increased in $\mathrm{db} / \mathrm{db}$ mice, and this effect was significantly reversed by Irb administration. (B) The serum TG and TC levels were increased in $\mathrm{db} / \mathrm{db}$ mice, and this effect was 
significantly reversed by Irb administration. (C) The liver function indexes ALT and AST were also increased in $\mathrm{db} / \mathrm{db}$ mice, but Irb treatment dramatically alleviated liver dysfunction. The values are expressed as the means $\pm \mathrm{SD}$ ( $\mathrm{n}=12$ per group). ${ }^{*} P<0.05$ versus $\mathrm{db} / \mathrm{m}$ mice, ${ }^{\#} P<0.05$ versus $\mathrm{db} / \mathrm{db}$ mice.

Figure 2: Irb alleviates the increased lipid content and pathological changes in the liver. (A and B) Liver TG, TC, and FFA levels were increased in $\mathrm{db} / \mathrm{db}$ mice, and this effect was significantly reversed by Irb administration. (C and D) Representative liver sections stained with Oil Red O and HE (200x). (E) The NAS score of the HE staining was increased in db/db mice but decreased in $\mathrm{db} / \mathrm{db}$ mice receiving Irb treatment. (F) Quantitative data for the percentage of oil deposits in the liver (Oil Red O-positive areas) show increased depositions in $\mathrm{db} / \mathrm{db}$ mice but decreased depositions in $\mathrm{db} / \mathrm{db}$ mice receiving Irb treatment. The values are presented as the means $\pm \mathrm{SD}$ (n=6-7 per group). ${ }^{*} P<0$.versus $\mathrm{db} / \mathrm{m}$ mice, ${ }^{\#} P<0.05$ versus $\mathrm{db} / \mathrm{db}$ mice.

Figure 3: Irb increases PPAR- $\gamma$ levels and activates the AMPK/Akt/mTOR signaling pathway. (A) Representative Western blot of PPAR- $\gamma$ expression in the liver. (B) Quantification of the expression levels of PPAR- $\gamma$ protein. (C) The expression levels of AMPK, p-AMPK, Akt, p-Akt, mTOR, and p-mTOR were assessed by Western blotting. (D) Densitometric analysis of the p-AMPK/AMPK, p-Akt/Akt, and p-mTOR/mTOR ratios. The data are derived from three separate experiments and are presented as the means \pm SD. ${ }^{*} P<0.05$ versus $\mathrm{db} / \mathrm{m}$ mice, ${ }^{\#} P<0.05$ versus $\mathrm{db} / \mathrm{db}$ mice.

Figure 4: Irb induces autophagy in the livers of $\mathrm{db} / \mathrm{db}$ mice. (A and B) Immunofluorescence and immunohistochemistry analysis of the autophagosome marker LC3B in liver tissue. The tissues were stained with an antibody against LC3B (red) and the nuclear dye DAPI (blue) (200x). (C and D) Quantification of the IF and IHC staining. (E) Representative Western blot of LC3B expression in the liver. (F) Quantification of the protein expression levels of LC3B. (G) Autophagosome formation was detected in hepatocytes by using TEM and was significantly increased after Irb treatment. Arrows indicate autophagosomes. The data are expressed as the means $\pm \mathrm{SD}$ of three independent experiments. ${ }^{*} P<0.05$ versus $\mathrm{db} / \mathrm{m}$ mice, ${ }^{\#} P<0.05$ versus $\mathrm{db} / \mathrm{db}$ mice.

Supplemental Table 1: The effects of Irb on body weight and tissue weight in female mice.

Supplemental Table 2: The effects of Irb on body weight and tissue weight in male mice.

Supplemental Figure 1: Irb improves the lipid content in the serum and liver tissue of the female and male $\mathrm{db} / \mathrm{db}$ mice. (A and B) The serum TG and TC levels were increased in female and male $\mathrm{db} / \mathrm{db}$ mice, and this effect was significantly reversed after Irb administration. (C and D) The liver TG and TC levels were increased in female and male $\mathrm{db} / \mathrm{db}$ mice, and this effect was significantly reversed after Irb administration. (E and F) The liver FFA was also increased in female and male $\mathrm{db} / \mathrm{db}$ mice, but Irb treatment dramatically reduces these levels. The values are presented as the means $\pm \mathrm{SD}$ (n=6 per group). ${ }^{*} P<0.05$ versus $\mathrm{db} / \mathrm{m}$ mice, ${ }^{\sharp} P<0.05$ versus $\mathrm{db} / \mathrm{db}$ mice. 
liver, brown fat and hearts of db/db mice. (A, C and E) The expression levels of leptin, LepR, and PPAR- $\alpha$ were assessed in the liver, brown fat and hearts by Western blotting. (B, D and F) Quantification of the protein expression levels of leptin, LepR, and PPAR- $\alpha$ in the liver, brown fat and heart. The data are derived from three separate experiments and are presented as the means \pm SD. ${ }^{*} P<0.05$ versus $\mathrm{db} / \mathrm{m}$ mice, ${ }^{\#} P<0.05$ versus $\mathrm{db} / \mathrm{db}$ mice.

\section{Discussion}

DM and NAFLD often occur in patients simultaneously and exert complex influences on each other. The purpose of this study was to determine the effect of Irb on hyperlipidemia and hepatic steatosis in type 2 diabetic $\mathrm{db} / \mathrm{db}$ mice. The results showed that Irb administration normalized most parameters of the lipid profile and resulted in a distinct improvement in the histopathological changes in the livers of $\mathrm{db} / \mathrm{db}$ mice. Consequently, Irb has beneficial therapeutic effects in alleviating hyperlipidemia and liver steatosis of $\mathrm{db} / \mathrm{db}$ mice, which might be associated with its ability to upregulate the expression of PPAR- $\gamma$, activate the AMPK/Akt/mTOR signaling pathway, and induce autophagy in diabetic $\mathrm{db} / \mathrm{db}$ mice livers.

Irb, as a unique subset of ARBs, is universally used in clinical practice and is also capable of activating PPAR- $\gamma$ [21-23]. Based on clinical trials and animal studies, Irb has been reported to have beneficial effects on ameliorating insulin resistance, reducing blood lipids, and preventing metabolic syndrome as well as protecting endothelial function via activation of PPAR- $\gamma[14,24-26]$. Moreover, the studies of Kato et al. [27] and Toblli et al. [28] showed that Irb evidently attenuated hepatic steatosis of FLS-ob/ob mice and obese Zucker rats, which was mediated by reducing the expression of SREBP1c and modulating inflammatory responses, respectively. In addition, a study from Kyoto University conducted by Rong et al. [1] revealed the efficacy of Irb in improving hypertriglyceridemia and reducing FFA concentrations, which was associated with the upregulation of the expression of PPAR- $\alpha$ and its target genes in the livers of obese Koletsky (fak/fak) rats. In contrast, the studies of Jia et al. [17] and den Besten et al. [29] demonstrated that astaxanthin and short-chain fatty acids derived from guar gum reduced hepatic lipids and protected against metabolic syndrome via the repression of PPAR- $\gamma$. However, our study showed that Irb treatment of $\mathrm{db} / \mathrm{db}$ mice reduced the associated weight gains in the body and liver, moderated food consumption, improved the lipid content in serum and liver tissue, and alleviated hepatic steatosis and liver dysfunction. This activity might be related to the upregulation of PPAR- $\gamma$ expression and the subsequent acceleration of lipogenesis and lipoprotein transport in the liver, thereby reducing lipotoxicity [30]. Furthermore, we also found that the protein expression of AMPK and p-AMPK was increased and that the expression of p-Akt was decreased when PPAR- $\gamma$ was activated. Therefore, Irb can regulate the expression of PPAR- $\gamma$, p-AMPK, and p-Akt. Further investigations are needed to determine whether Irb simultaneously activates the three proteins or if the three proteins are mutually influenced following Irb treatment. In addition, Irb decreased the leptin levels and increased PPAR- $\alpha$ expression in the livers, 
brown fats and hearts of $\mathrm{db} / \mathrm{db}$ mice, which contributed to the amelioration of leptin resistance and the stimulation of lipid metabolism in tissues, which is consistent with previous studies $[1,12]$.

Autophagy, a regulated cellular mechanism that degrades long-lived proteins and intracellular organelles, has been recently confirmed to improve lipid metabolism and hepatic steatosis [3,8,31]. The studies of Singh et al. [8] and Lin et al. [32] demonstrated that autophagy regulates lipid content and that the manipulation of autophagic activity influences fat accumulation in the liver. Ezaki et al. [33] reported that liver autophagy contributes to the maintenance of blood glucose levels. Taken together, these studies reveal that autophagy participates in metabolic diseases and provide a new method for preventing metabolic syndrome and its related pathologies. However, Zhang et al. [4] investigated the effect of autophagy and endoplasmic reticulum stress on diabetic mice. They revealed that the LC3BII protein levels were decreased at 8 months in diabetic OVE26 mice and that the suppression of autophagy in the liver was positively associated with the development of steatohepatitis. AMPK is involved in intracellular energy metabolism and affects glucose transport and $\beta$-oxidation in the liver [34-37]. Jimenez et al. [5] found that the expression levels of AMPK and PPAR- $\gamma$ were significantly reduced in $\mathrm{db} / \mathrm{db}$ mouse livers and that curcumin increased the expression of these proteins, thereby playing a beneficial role in treating the complications of type 2 diabetes. The study results of Huang et al. [38] showed that autophagy in SK-HEP-1 human hepatic cancer cells was induced by kaempferol via increasing p-AMPK levels, decreasing p-Akt levels, and activating the AMPK/Akt/mTOR signaling pathway. Foglio et al. [39] also revealed that the activation of autophagy in a murine model of myocardial infarction is most likely due to modulation of AMPK/Akt/mTOR signaling. The AMPK/Akt/mTOR signaling pathway has been reported to be an important regulator of autophagy [40-43]. Thus, whether Irb-induced autophagy in the diabetic livers of $\mathrm{db} / \mathrm{db}$ mice is associated with either PPAR- $\gamma$, AMPK, and Akt expression or activation of the AMPK/Akt/mTOR signaling pathway remains to be elucidated. In our study, we also found that the expression levels of PPAR- $\gamma$ and AMPK were significantly decreased and that the expression of p-Akt was increased in $\mathrm{db} / \mathrm{db}$ mice, which is consistent with the results of previous studies. Interestingly, the LC3B expression detected by IHC and IF staining, the LC3BII/I ratio and autophagosomes observed by TEM were reduced in $\mathrm{db} / \mathrm{db}$ mice compared with $\mathrm{db} / \mathrm{m}$ mice, whereas Irb treatment increased the LC3B expression, LC3BII/I ratio, and autophagosome number by activating PPAR- $\gamma$ and p-AMPK and inhibiting p-Akt and p-mTOR. In our opinion, Irb-induced autophagy may be related to the upregulation of PPAR- $\gamma$ and the activation of the AMPK/Akt/mTOR signaling pathway.

In the present study, we demonstrated the beneficial effects of Irb on hyperlipidemia and hepatic steatosis in diabetic $\mathrm{db} / \mathrm{db}$ mice. However, there are some limitations to our study. First, although the diabetic $\mathrm{db} / \mathrm{db}$ mouse model exhibits many metabolic phenotypes that are observed in patients, the relevance to actual clinical conditions may be limited. Second, our study demonstrated that Irb could induce liver autophagy 
as a PPAR- $\gamma$ agonist. However, the precise role of PPAR- $\gamma$ in autophagy has not been definitively verified. Moreover, the study of Seok et al. [44] revealed that the FXR-CREB axis regulated autophagy and that the results of Xiao et al. [3] showed that activation of ERK1/2 ameliorated liver steatosis of $\mathrm{db} / \mathrm{db}$ mice via the stimulation of ATG7-dependent autophagy. Therefore, in future work, we will focus on studying autophagy-related proteins and signaling pathways by employing PPAR- $\gamma$ and autophagy-specific inhibitors or using an Ad-GFP-LC3-constructed mouse model to further confirm the effects of Irb on liver autophagy.

\section{Conclusions}

In summary, Irb alleviated hyperlipidemia and liver steatosis in type 2 diabetic $\mathrm{db} / \mathrm{db}$ mice. The molecular mechanisms that drive the therapeutic effects of Irb may involve the upregulation of PPAR- $\gamma$, activation of the AMPK/Akt/mTOR signaling pathway, and liver autophagy. Our study may demonstrate the generation of therapeutic agents useful in improving lipid metabolism and hepatic steatosis.

\section{Acknowledgement}

We acknowledge the financial support from the National Science Foundation of China (No. 81573729 and 81373808), the Science and Technology Planning Project of Guangdong Province (No. 2013B021800149, 2014A020210011, 2015A020211012), and the Science and Technology Planning Project of Guangzhou (No. 201510010137). We would like to thank Lei Gao, Hong Li, and Dexiu Chen for their suggestions. 


\section{References}

[1] X. Rong, Y. Li, K. Ebihara, M. Zhao, T. Kusakabe, T. Tomita, M. Murray, K. Nakao, Irbesartan treatment up-regulates hepatic expression of PPARalpha and its target genes in obese Koletsky $(\mathrm{fa}(\mathrm{k}) / \mathrm{fa}(\mathrm{k}))$ rats: a link to amelioration of hypertriglyceridaemia, Br. J. Pharmacol. 160 (2010) 1796-1807.

[2] N.H. Parikh, P.K. Parikh, C. Kothari, Indigenous plant medicines for health care: treatment of diabetes mellitus and hyperlipidemia, Chin. J. Nat. Med. 12 (2014) 335-344.

[3] Y. Xiao, H. Liu, J. Yu, Z. Zhao, F. Xiao, T. Xia, C. Wang, K. Li, J. Deng, Y. Guo, S. Chen, Y. Chen, F. Guo, Activation of ERK1/2 ameliorates liver steatosis in leptin receptor-deficient $(\mathrm{db} / \mathrm{db})$ mice via stimulating ATG7-dependent autophagy, Diabetes 65 (2016) 393-405.

[4] Q. Zhang, Y. Li, T. Liang, X. Lu, C. Zhang, X. Liu, X. Jiang, R.C. Martin, M. Cheng, L. Cai, ER stress and autophagy dysfunction contribute to fatty liver in diabetic mice, Int. J. Biol. Sci. 11 (2015) 559-568.

[5] L.M. Jimenez-Flores, S. Lopez-Briones, M.H. Macias-Cervantes, J. Ramirez-Emiliano, V. Perez-Vazquez, A PPARgamma, NF-kappaB and AMPK-dependent mechanism may be involved in the beneficial effects of curcumin in the diabetic db/db mice liver, Molecules 19 (2014) 8289-8302.

[6] I. Taguchi, S. Toyoda, K. Takano, T. Arikawa, M. Kikuchi, M. Ogawa, S. Abe, K. Node, T. Inoue, Irbesartan, an angiotensin receptor blocker, exhibits metabolic, anti-inflammatory and antioxidative effects in patients with high-risk hypertension, Hypertens. Res. 36 (2013) 608-613. 
[7] Y. Zhang, M.L. Chen, Y. Zhou, L. Yi, Y.X. Gao, L. Ran, S.H. Chen, T. Zhang, X. Zhou, D. Zou, B. Wu, Y. Wu, H. Chang, J.D. Zhu, Q.Y. Zhang, M.T. Mi, Resveratrol improves hepatic steatosis by inducing autophagy through the cAMP signaling pathway, Mol. Nutr. Food Res. 59 (2015) 1443-1457.

[8] R. Singh, S. Kaushik, Y. Wang, Y. Xiang, I. Novak, M. Komatsu, K. Tanaka, A.M. Cuervo, M.J. Czaja, Autophagy regulates lipid metabolism, Nature 458 (2009) 1131-1135.

[9] A. Shiota, M. Shimabukuro, D. Fukuda, T. Soeki, H. Sato, E. Uematsu, Y. Hirata, H. Kurobe, N. Maeda, H. Sakaue, H. Masuzaki, I. Shimomura, M. Sata, Telmisartan ameliorates insulin sensitivity by activating the AMPK/SIRT1 pathway in skeletal muscle of obese $\mathrm{db} / \mathrm{db}$ mice, Cardiovasc. Diabetol. 11 (2012) 139.

[10] X. Qiang, L. Xu, M. Zhang, P. Zhang, Y. Wang, Y. Wang, Z. Zhao, H. Chen, X. Liu, Y. Zhang, Demethyleneberberine attenuates non-alcoholic fatty liver disease with activation of AMPK and inhibition of oxidative stress, Biochem. Biophys. Res. Commun. 472 (2016) 603-609.

[11] F. Dol, G. Martin, B. Staels, A.M. Mares, C. Cazaubon, D. Nisato, J.P. Bidouard, P. Janiak, P. Schaeffer, J.M. Herbert, Angiotensin AT1 receptor antagonist irbesartan decreases lesion size, chemokine expression, and macrophage accumulation in apolipoprotein E-deficient mice, J. Cardiovasc. Pharmacol. 38 (2001) 395-405.

[12] A. Maeda, K. Tamura, H. Wakui, M. Ohsawa, K. Azushima, K. Uneda, R. 
Kobayashi, Y. Tsurumi-Ikeya, T. Kanaoka, T. Dejima, K. Ohki, S. Haku, A.

Yamashita, S. Umemura, Effects of Ang II receptor blocker irbesartan on adipose tissue function in mice with metabolic disorders, Int. J. Med. Sci. 11 (2014) 646-651.

[13] P. Le Corvoisier, C. Adamy, L. Sambin, B. Crozatier, A. Berdeaux, J.B. Michel, L. Hittinger, J. Su, The cardiac renin-angiotensin system is responsible for high-salt diet-induced left ventricular hypertrophy in mice, Eur. J. Heart Fail. 12 (2010) 1171-1178.

[14] M. Iwai, H. Kanno, I. Senba, H. Nakaoka, T. Moritani, M. Horiuchi, Irbesartan increased PPARgamma activity in vivo in white adipose tissue of atherosclerotic mice and improved adipose tissue dysfunction, Biochem. Biophys. Res. Commun. 406 (2011) 123-126.

[15] M. Iwai, H. Sone, H. Kanno, T. Moritani, M. Horiuchi, Reciprocal regulation of cholesterol excretion in apolipoprotein E-null mice by angiotensin II type 1 and type 2 receptor deficiency, Life Sci. 92 (2013) 276-281.

[16] D.E. Kleiner, E.M. Brunt, M. Van Natta, C. Behling, M.J. Contos, O.W. Cummings, L.D. Ferrell, Y.C. Liu, M.S. Torbenson, A. Unalp-Arida, M. Yeh, A.J. McCullough, A.J. Sanyal, Design and validation of a histological scoring system for nonalcoholic fatty liver disease, Hepatology 41 (2005) 1313-1321.

[17] Y. Jia, C. Wu, J. Kim, B. Kim, S.J. Lee, Astaxanthin reduces hepatic lipid accumulations in high-fat-fed C57BL/6J mice via activation of peroxisome proliferator-activated receptor (PPAR) alpha and inhibition of PPAR gamma 
and Akt, J. Nutr. Biochem. 28 (2016) 9-18.

[18] J. Xiao, R. Guo, M.L. Fung, E.C. Liong, R.C. Chang, Y.P. Ching, G.L. Tipoe, Garlic-derived S-allylmercaptocysteine ameliorates nonalcoholic fatty liver disease in a rat model through inhibition of apoptosis and enhancing autophagy, Evid. Based Complement. Alternat. Med. 2013 (2013) 642920.

[19] R. Clasen, M. Schupp, A. Foryst-Ludwig, C. Sprang, M. Clemenz, M. Krikov, C. Thone-Reineke, T. Unger, U. Kintscher, PPARgamma-activating angiotensin type-1 receptor blockers induce adiponectin, Hypertension 46 (2005) 137-143.

[20] J. Ge, J.J. Miao, X.Y. Sun, J.Y. Yu, Huangkui capsule, an extract from Abelmoschus manihot (L.) medic, improves diabetic nephropathy via activating peroxisome proliferator-activated receptor (PPAR)-alpha/gamma and attenuating endoplasmic reticulum stress in rats, J. Ethnopharmacol. 189 (2016) 238-249.

[21] I.T. Abdel-Raheem, G.A. Omran, M.A. Katary, Irbesartan, an angiotensin II receptor antagonist, with selective PPAR-gamma-modulating activity improves function and structure of chemotherapy-damaged ovaries in rats, Fundam. Clin. Pharmacol. 29 (2015) 286-298.

[22] Z.Z. Zhang, Q.H. Shang, H.Y. Jin, B. Song, G.Y. Oudit, L. Lu, T. Zhou, Y.L. Xu, P.J. Gao, D.L. Zhu, J.M. Penninger, J.C. Zhong, Cardiac protective effects of irbesartan via the PPAR-gamma signaling pathway in angiotensin-converting enzyme 2-deficient mice, J. Transl. Med. 11 (2013) 
[23] H. Kusunoki, Y. Taniyama, H. Rakugi, R. Morishita, Cardiac and renal protective effects of irbesartan via peroxisome proliferator-activated receptorgamma-hepatocyte growth factor pathway independent of angiotensin II Type 1a receptor blockade in mouse model of salt-sensitive hypertension, J. Am. Heart Assoc. 2 (2013) e000103.

[24] S. Takai, D. Jin, M. Miyazaki, Irbesartan prevents metabolic syndrome in rats via activation of peroxisome proliferator-activated receptor gamma, J. Pharmacol. Sci. 116 (2011) 309-315.

[25] M. Shimamura, H. Nakagami, T. Shimosato, T. Moritani, F. Nakagami, M.K. Osako, T. Miyake, H. Koriyama, H. Shimizu, R. Morishita, Irbesartan improves endothelial dysfunction, abnormal lipid profile, proteinuria and liver dysfunction in Zucker diabetic fatty rats independent of glucose and insulin levels, Exp. Ther. Med. 2 (2011) 957-961.

[26] A. Chatterjee, H. Kusunoki, Y. Taniyama, H. Rakugi, R. Morishita, Improvement of metabolic syndrome by irbesartan via the PPARgamma/HGF pathway in apolipoprotein E knockout mice, Biomed. Rep. 1 (2013) 65-70.

[27] J. Kato, M. Koda, M. Kishina, S. Tokunaga, T. Matono, T. Sugihara, M. Ueki, Y. Murawaki, Therapeutic effects of angiotensin II type 1 receptor blocker, irbesartan, on non-alcoholic steatohepatitis using FLS-ob/ob male mice, Int. J. Mol. Med. 30 (2012) 107-113.

[28] J.E. Toblli, M.C. Munoz, G. Cao, J. Mella, L. Pereyra, R. Mastai, ACE 
inhibition and AT1 receptor blockade prevent fatty liver and fibrosis in obese Zucker rats, Obesity (Silver Spring) 16 (2008) 770-776.

[29] G. den Besten, A. Gerding, T.H. van Dijk, J. Ciapaite, A. Bleeker, K. van Eunen, R. Havinga, A.K. Groen, D.J. Reijngoud, B.M. Bakker, Protection against the metabolic syndrome by guar gum-derived short-chain fatty acids depends on peroxisome proliferator-activated receptor gamma and glucagon-like peptide-1, PLoS One 10 (2015) e0136364.

[30] M. Gu, Y. Zhang, S. Fan, X. Ding, G. Ji, C. Huang, Extracts of Rhizoma polygonati odorati prevent high-fat diet-induced metabolic disorders in C57BL/6 mice, PLoS One 8 (2013) e81724.

[31] R. Singh, A.M. Cuervo, Lipophagy: connecting autophagy and lipid metabolism, Int. J. Cell Biol. 2012 (2012) 282041.

[32] C.W. Lin, H. Zhang, M. Li, X. Xiong, X. Chen, X. Chen, X.C. Dong, X.M. Yin, Pharmacological promotion of autophagy alleviates steatosis and injury in alcoholic and non-alcoholic fatty liver conditions in mice, J. Hepatol. 58 (2013) 993-999.

J. Ezaki, N. Matsumoto, M. Takeda-Ezaki, M. Komatsu, K. Takahashi, Y. Hiraoka, H. Taka, T. Fujimura, K. Takehana, M. Yoshida, J. Iwata, I. Tanida, N. Furuya, D.M. Zheng, N. Tada, K. Tanaka, E. Kominami, T. Ueno, Liver autophagy contributes to the maintenance of blood glucose and amino acid levels, Autophagy 7 (2011) 727-736.

[34] Y. Peng, J. Liu, L. Shi, Y. Tang, D. Gao, J. Long, J. Liu, Mitochondrial 
dysfunction precedes depression of AMPK/AKT signaling in insulin resistance induced by high glucose in primary cortical neurons, J. Neurochem. 137 (2016) 701-713.

[35] Y.H. Choi, J.K. Bae, H.S. Chae, Y.M. Kim, Y. Sreymom, L. Han, H.Y. Jang, Y.W. Chin, Alpha-mangostin regulates hepatic steatosis and obesity through SirT1-AMPK and PPARgamma pathways in high-fat diet-induced obese mice, J. Agric. Food Chem. 63 (2015) 8399-8406.

[36] Y. Wang, X. Li, Y. Guo, L. Chan, X. Guan, Alpha-lipoic acid increases energy expenditure by enhancing adenosine monophosphate-activated protein kinase-peroxisome proliferator-activated receptor-gamma coactivator-1alpha signaling in the skeletal muscle of aged mice, Metabolism 59 (2010) 967-976.

[37] F. Yao, M. Zhang, L. Chen, 5'-Monophosphate-activated protein kinase (AMPK) improves autophagic activity in diabetes and diabetic complications, Acta Pharm. Sin. B 6 (2016) 20-25.

[38] W.W. Huang, S.C. Tsai, S.F. Peng, M.W. Lin, J.H. Chiang, Y.J. Chiu, S. Fushiya, M.T. Tseng, J.S. Yang, Kaempferol induces autophagy through AMPK and AKT signaling molecules and causes G2/M arrest via downregulation of CDK1/cyclin B in SK-HEP-1 human hepatic cancer cells, Int. J. Oncol. 42 (2013) 2069-2077.

[39] E. Foglio, G. Puddighinu, A. Germani, M.A. Russo, F. Limana, HMGB1 inhibits apoptosis following MI and induces autophagy via mTORC1 inhibition, J. Cell Physiol. (2016) doi: 10.1002/jcp.25576. 
[40] P. Duan, C. Hu, C. Quan, T. Yu, W. Zhou, M. Yuan, Y. Shi, K. Yang, 4-Nonylphenol induces apoptosis, autophagy and necrosis in Sertoli cells: involvement of ROS-mediated AMPK/AKT-mTOR and JNK pathways, Toxicology 341-343 (2016) 28-40.

[41] H.C. Furlong, M.R. Stampfli, A.M. Gannon, W.G. Foster, Cigarette smoke exposure triggers the autophagic cascade via activation of the AMPK pathway in mice, Biol. Reprod. 93 (2015) 93.

[42] K.M. Mellor, M.E. Reichelt, L.M. Delbridge, Autophagy anomalies in the diabetic myocardium, Autophagy 7 (2011) 1263-1267.

[43] Y. Zhang, J. Ren, Autophagy in ALDH2-elicited cardioprotection against ischemic heart disease: slayer or savior?, Autophagy 6 (2010) 1212-1213.

[44] S. Seok, T. Fu, S.E. Choi, Y. Li, R. Zhu, S. Kumar, X. Sun, G. Yoon, Y. Kang, W. Zhong, J. Ma, B. Kemper, J.K. Kemper, Transcriptional regulation of autophagy by an FXR-CREB axis, Nature 516 (2014) 108-111. 

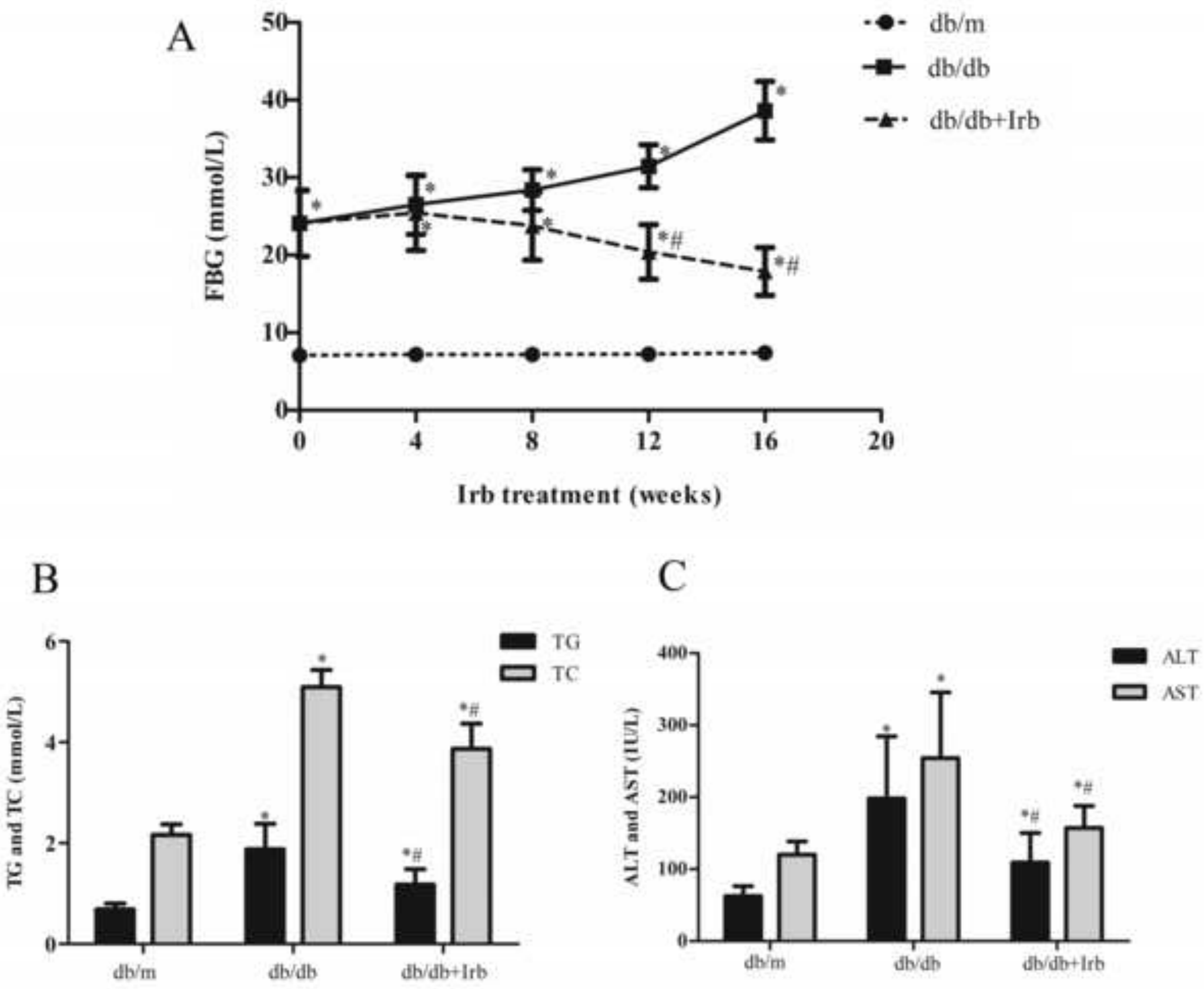

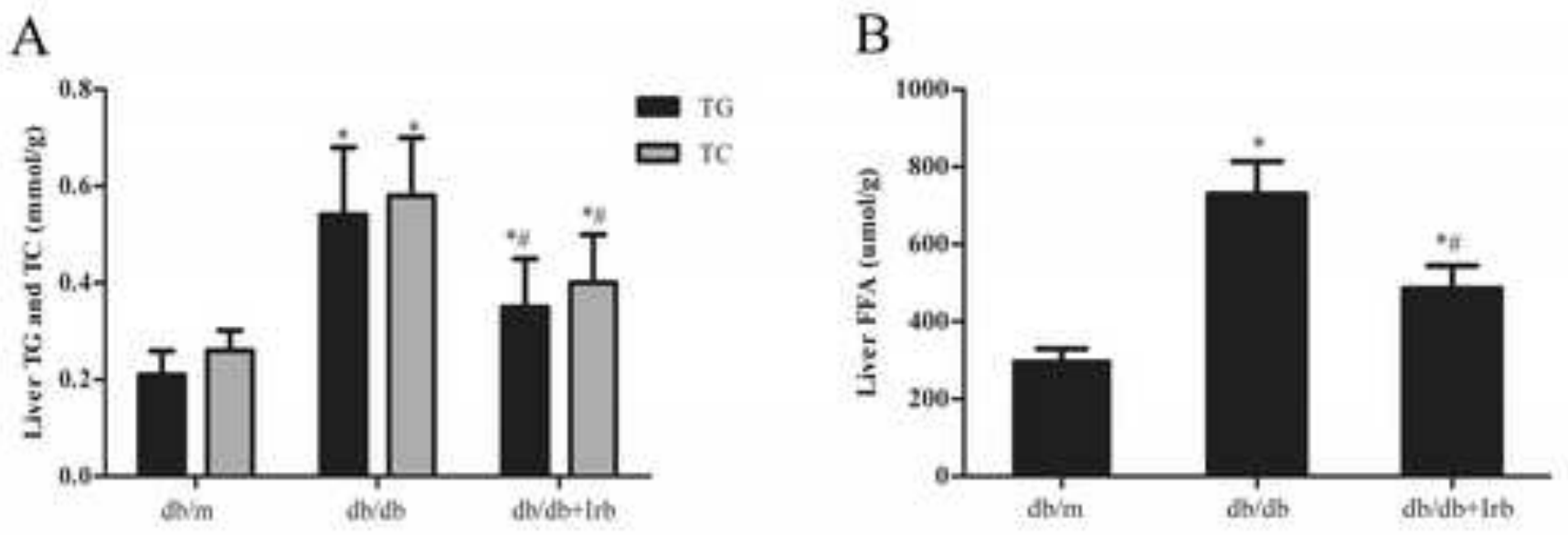

C

HE

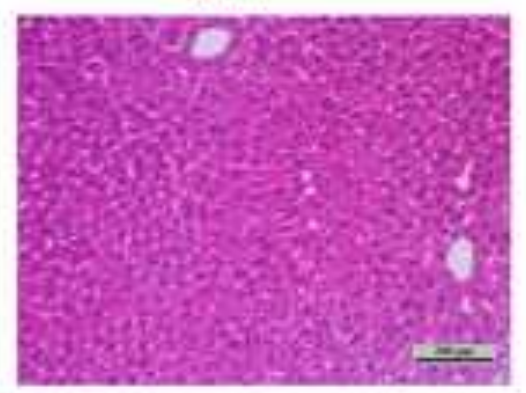

D

$\mathrm{db} / \mathrm{m}$

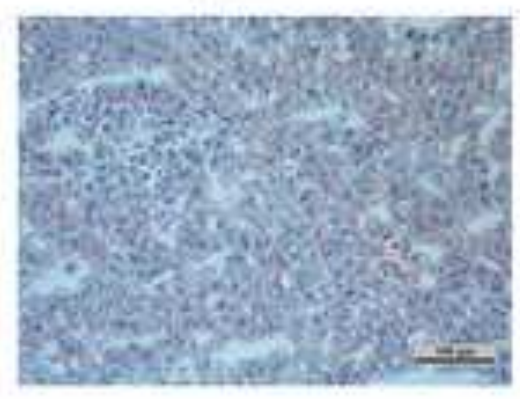

dbidb

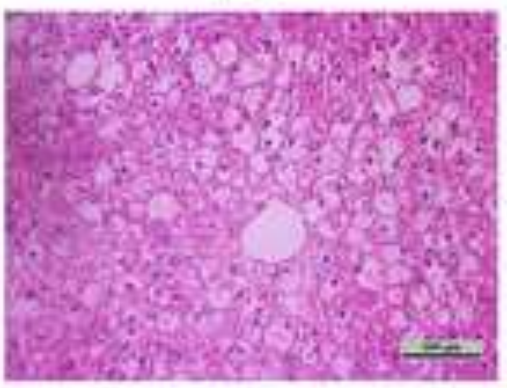

dbidb

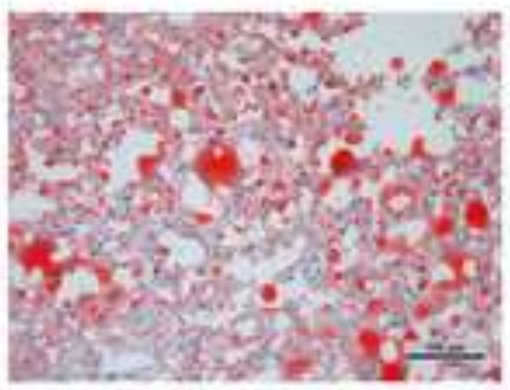

$\mathrm{db} / \mathrm{db}+\mathrm{Irb}$

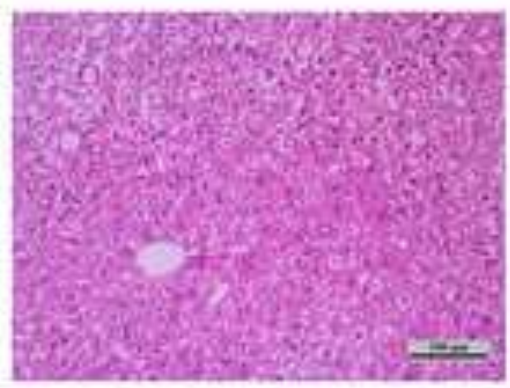

db/db+irb

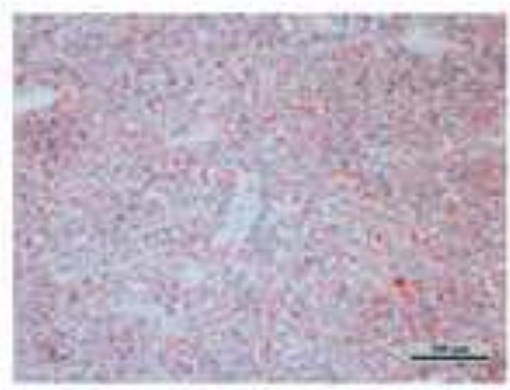

E

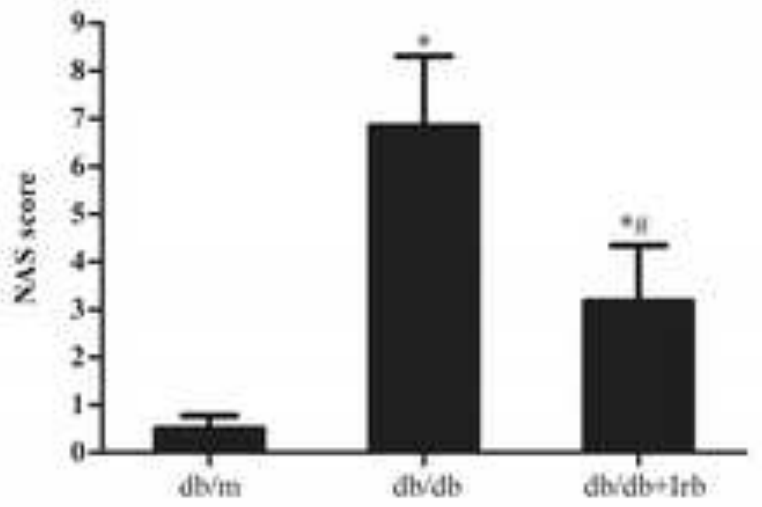

F

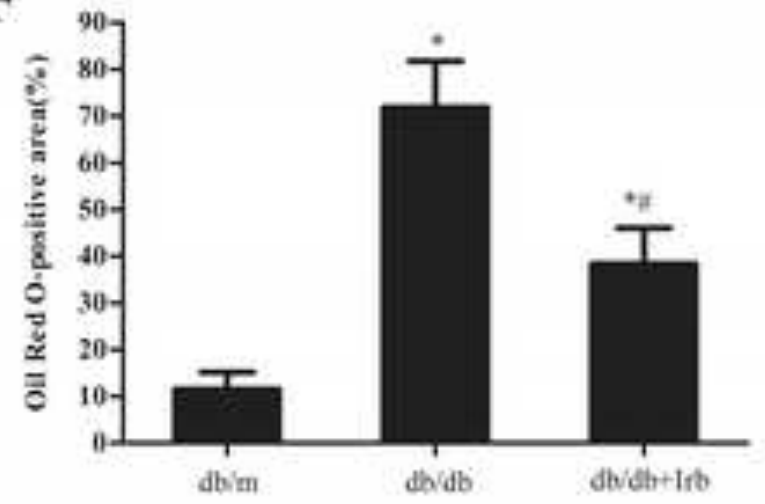




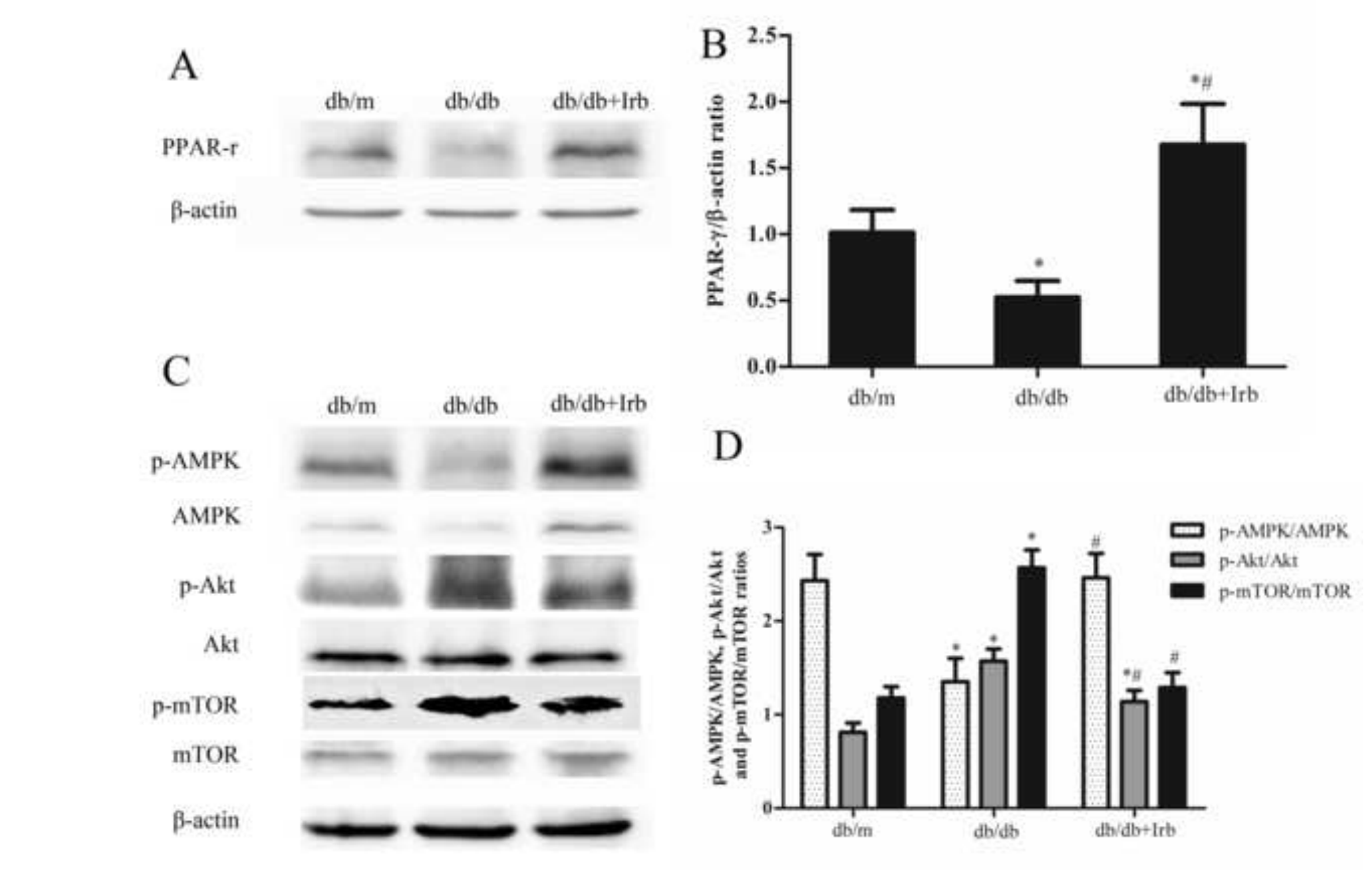

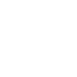

.


B

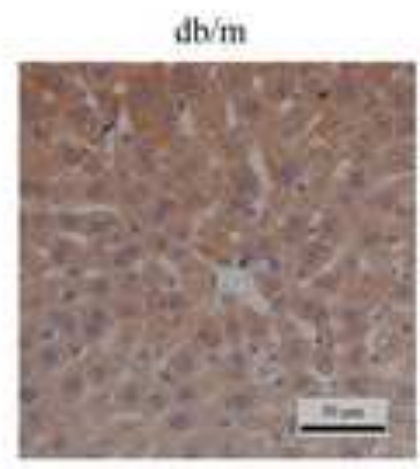

C

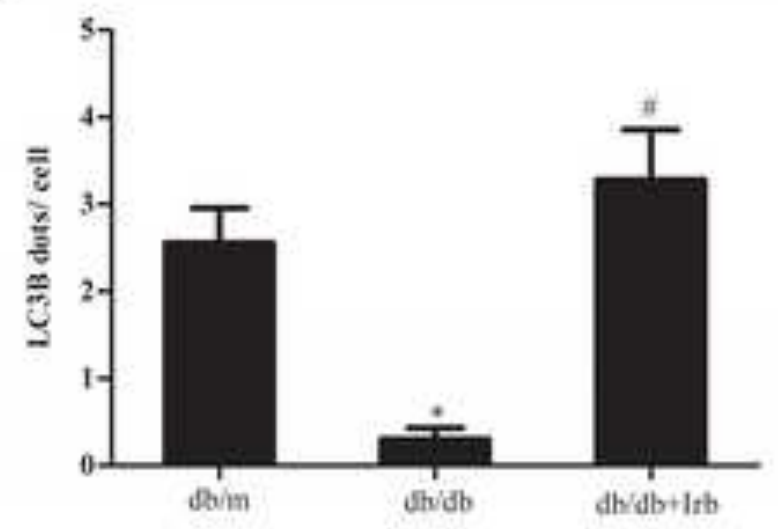

E

$d b / m \quad d b / d b \quad d b / d b+i b$

\section{LC3-1}

L.C3.II

B-actin

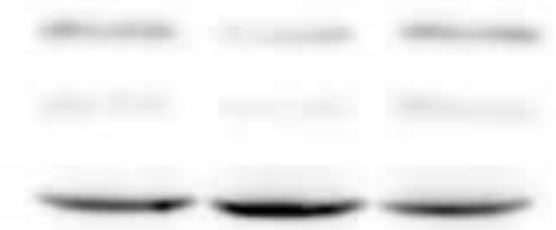

$\mathrm{db} / \mathrm{db}$

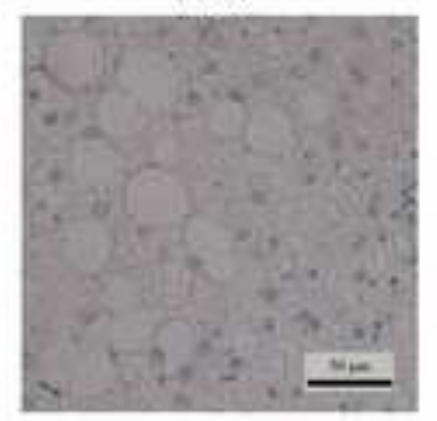

D
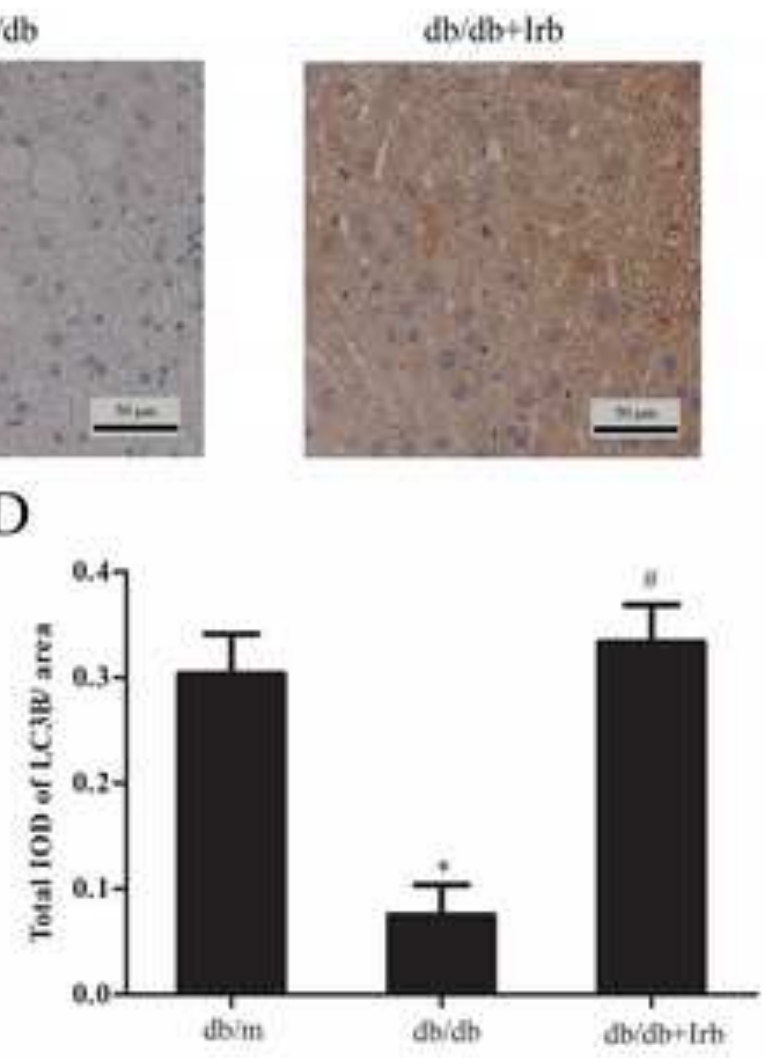

F

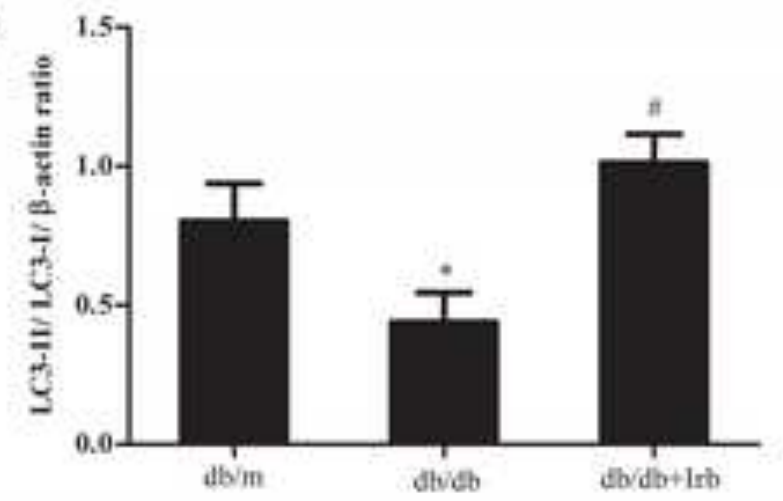

G

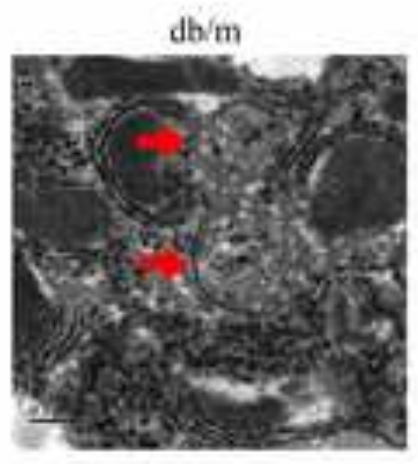

$\mathrm{db} / \mathrm{db}$

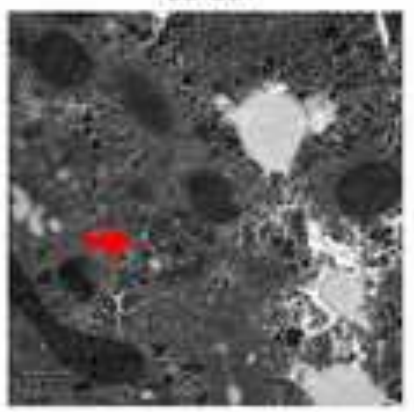

$\mathrm{db} / \mathrm{db}+\mathrm{Irb}$

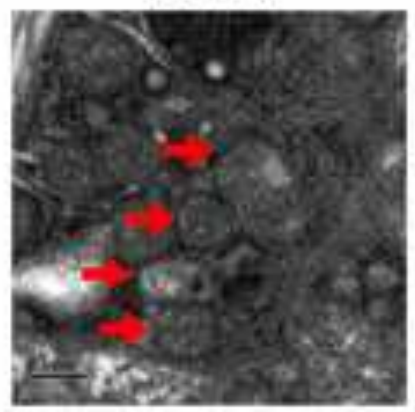

